\title{
UniStatus - a cross-sectional study on the contamination of uniforms in the Danish ambulance service
}

Heidi Storm Vikke* and Matthias Giebner

\begin{abstract}
Background: Patients are at risk of contracting infections due to the presence of disease-causing microorganisms that can be transmitted from the medical staff's uniforms to the patient. The dual purpose of this study was to examine the contamination level of the uniforms worn by ambulance staff after a shift and to test the effect of washing of the uniform with and without a detergent containing acetic peroxide.

Methods: This was a cross-sectional study in which 30 ambulance staff uniforms were randomly selected for inclusion and divided into two groups. Before washing, 90 prints were performed with specific agar plates to determine bacterial contamination and to establish the prevalence of a variety of microorganisms. Group A uniforms were washed with a detergent without acetic peroxide; Group B uniforms were washed with a detergent containing acetic peroxide.
\end{abstract}

Results: Before washing, the 90 prints had an average colony-forming units (CFU) of potentially pathogenic bacteria of 68.89 per $25 \mathrm{~cm}^{2}$ and a prevalence of: E. coli and Pseudomonas 0\%, Bacillus cereus 27.78\% (Cl 95\% \pm 9.80 ), Clostridium and Enterococcus 2.22\% (Cl 95\% \pm 1.96 ), Staphylococcus aureus 21.11\% (Cl 95\% \pm 7.80 ). After washing, CFU was reduced to 3.09 ( $\mathrm{Cl} 95 \% \pm 5.04)$ per $25 \mathrm{~cm}^{2}$ in Group A and to 1.47 (Cl 95\% \pm 4.77) per $25 \mathrm{~cm}^{2}$ in Group B. The prevalence of specific bacteria in either group was 0\%, except for $S$. aureus which had a prevalence rate of 4.40\% (Cl 95\% \pm 6.10 ) in Group A. The difference between the contamination degrees of the two groups was not significant in either test $(p>0.05)$.

Conclusion: Potentially pathogenic bacteria are detectable on ambulance staff uniforms when a shift ends. Optimal prevention of bacterial infection may be achieved by daily changing, washing at a minimum of 60 degrees Celsius and use of a detergent containing acetic peroxide.

Keywords: Prehospital, Uniform, Hygiene, Bacterial contamination, Infection control

\section{Background}

National and international prehospital staff have an interest in obtaining thorough knowledge about the contamination level of prehospital uniforms and, thus, about the risk of infection to which patients are exposed in the prehospital setting. Several international studies have examined various aspects related to health staff uniforms including, among others, the presence of methicillinresistant Staphylococcus aureus (MRSA) [1,2], the importance of the washing procedure for microbiological reduction [3] and the hygiene culture among health

\footnotetext{
* Correspondence: hevi@falck.dk

Medical department, Falck Denmark A/S, 6000 Kolding, Denmark
}

professionals [4]. These studies, however, have all concerned hospital or home care staff.

Studies have demonstrated that uniforms in healthcare services become contaminated with microorganisms, among others through the staff's contact with patients [4]. Thus, a clinical study demonstrated the spreading of infection from the uniform to the patient and the transmission of infection from patient to uniform and then to another patient [5]. Several other studies have also shown that there is a risk of transmitting bacteria by direct contact [6]. This indicates that uniforms in prehospital healthcare services may also constitute a source of infection and thus a health risk for patients. 
In the Region of Southern Denmark, more than 100,000 patients are transported by ambulances annually (quote by: Niels Thorup, Regional Director, Falck Denmark A/S, Region of Southern Denmark, 2014). The staff in these ambulances carries standardized uniforms consisting of a jacket and a pair of trousers that are washed in accordance with instructions based on an accredited standard. Presumably, the washing procedure reduces the number and types of microorganisms on clothes after washing [3]. However, our literature search revealed no evidence of the exact significance of washing on daily practice in Danish prehospital settings.

Furthermore, several studies show that hospital uniforms can be contaminated with microorganisms $[1,7,8]$. The most commonly occurring microorganisms are $S$. aureus and Enterococcus, among several others [1,4]. Still, our literature search produced no studies examining which microorganisms are involved in the bacteriological contamination of staff uniforms in Danish ambulance services.

We therefore examined the extent to which the ambulance staff uniforms are contaminated with potentially pathogenic microorganisms before washing. Specifically, we examined the potentially pathogenic bacterial contamination and the prevalence rate for E. coli, B. cereus, Clostridium, S. aureus, Enterococcus and Pseudomonas. We also examined to which extent the bacterial contamination was reduced through use of a washing detergent with and without acetic peroxide. All uniforms were washed at 60 degrees Celsius and tumble-dried for 1 hour.

\section{Methods}

A cross-sectional study was carried out at a random number of selected ambulance stations in the Region of Southern and Central Denmark. In accordance with standard procedures, uniforms were washed locally in the stations where the staff is employed. Practical sampling was applied, and the collected uniforms had all been used in the ambulance service within the past 24 hours. The collection took place over a period of 7 days. The staff was not blinded as they are usually responsible for the washing of uniforms.

A total of 30 ambulance staff uniforms were selected randomly after they had been worn during a shift lasting a minimum of 8 hours. Before washing, all 30 uniforms were contact-printed with specific $25 \mathrm{~cm}^{2}$ agar plates or inoculated using swab sticks for bacterial mapping.

In order to determine the level of potentially pathogenic contamination, blood agar plates were used, and the samples were incubated at $37 \pm 1$ degrees Celsius for $24 \pm 3$ hours. To determine the prevalence of $B$. cereus, blood agar plates were used and subsequently incubated at $30 \pm 1$ degrees Celsius for 24 hours. S. aureus prevalence was determined using rabbit plasma fibrinogen (RPF) agar plates which were incubated at $37 \pm 1$ degrees
Celsius for 18-24 hours and, if necessary +24 hours. For the examination of Enterococcus prevalence, we used Slanetz agar and an incubation temperature of $44 \pm 1$ degrees Celsius for $48 \pm 4$ hours. The Clostridium prevalence was examined with tryptose sulphite cycloserine (TSC) at an incubation temperature of $37 \pm 1$ degrees Celsius for $24 \pm 3$ hours. The Pseudomonas prevalence was examined with rapid Pseudomonas agar at an incubation temperature of 36 degrees Celsius for 22-33 hours. Finally, the E. coli prevalence was examined by Tryptone Bile X-glucornide (TBX) agar at an incubation temperature of $44 \pm 1$ degrees Celsius for $21 \pm 3$ hours. Table 1 presents the laboratory methods used.

Prints were obtained from the torso, the thighs and the wrist area which previous studies have shown are the most contaminated locations on healthcare uniforms $[4,9]$.

\section{Random selection of the intervention groups}

The uniforms were randomly selected for washing and drying in two different groups before prints were taken with agar plates. After the prints were taken, the uniforms in Group A were washed in a domestic washing machine at 60 degrees Celsius with the Tex-Power 350 detergent that does not contain acetic peroxide. The uniforms were then dried in a domestic tumble dryer at medium heat for 60 minutes. The uniforms in Group B were also washed in a domestic washing machine at 60 degrees Celsius, but with the Tex Des 60 detergent which does contain acetic peroxide. The uniforms were then dried at medium heat in a domestic tumble dryer for 60 minutes. After washing and drying, specific agar prints were taken again from all uniforms, precisely as before the washing.

All agar prints were collected locally in the participating ambulance stations. The samples were then stored at $<5$ degrees Celsius during transportation to the laboratory. Incubation and laboratory analysis were performed in accordance with a DANAK-accredited method (quote by: Marianne Sloth Johansen, AnalyTech A/S, Denmark, 2014). The laboratory specified the results as number of CFU per $25 \mathrm{~cm}^{2}$.

\section{Statistical analysis}

Descriptive statistics were performed. The overall level of potentially pathogenic contamination before and after washing is given by mean, standard deviation (SD), minimum and maximum values and reported with a $95 \%$ confidence interval (CI). To test the difference in reduction of mean CFU, Student's t-test was performed with an alpha $=0.05$. The prevalence of each specific bacterium before and after washing was presented with a number of positive prints associated with a percentage with CI 95\%. The chi-squared test was used to test for significant difference $(\mathrm{alpha}=0.05)$ between the prevalence of 
Table 1 Laboratory methods

\begin{tabular}{lll}
\hline Bacterial focus & Agar plate/swab stick & Incubation time/temperature \\
\hline Potentially pathogenic bacteria & Blood agar & $37 \pm 1$ degrees Celsius for $24 \pm 3$ hours \\
B. cereus & Blood agar & $30 \pm 1$ degrees Celsius for 24 hours \\
S. aureus & RPF agar & $37 \pm 1$ degrees Celsius for $18-24$ hours* \\
Enterococcus & Slanetz agar & $44 \pm 1$ degrees Celsius for $48 \pm 4$ hours \\
Clostridium & TSC & $37 \pm 1$ degrees Celsius for $24 \pm 3$ hours \\
Pseudomonas & Rapid Pseudomonas agar & 36 degrees Celsius for $22-33$ hours \\
E-coli & TBX agar & $44 \pm 1$ degrees Celsius for $21 \pm 3$ hours \\
\hline
\end{tabular}

*If necessary, an additional 24 hours.

the chosen bacteria in the two groups. Furthermore, proportion tests were performed to test for significant differences in the percentage reduction among and between the groups. Our data were not $100 \%$ normally distributed; we therefore tested our results of the Student's t-test with a non-parametric test which showed similarity.

\section{Ethical considerations}

The study is exempted from ethical approval since it neither involved humans nor animals. To ensure that the study complied with normal standards and instructions, all uniforms were washed after they had been tested, i.e. before they were used in emergency medical services again.

\section{Results}

\section{Potentially pathogenic contamination level}

The potentially pathogenic contamination per uniform print before washing in Group A and Group B varied from 0 to $200 \mathrm{CFU}$ per $25 \mathrm{~cm}^{2}$. A total of 90 uniform prints were contaminated with an average of $68.89 \mathrm{CFU}$ per $25 \mathrm{~cm}^{2}(\mathrm{SD} \pm 65.83)$.

\section{Potentially pathogenic contamination level before and after washing}

Before washing, the average potentially pathogenic contamination level for the 45 selected uniform prints in Group A was $82.49 \mathrm{CFU}$ per $25 \mathrm{~cm}^{2}(\mathrm{SD} \pm 65.21)$. For the prints in Group B, the average potentially pathogenic contamination level was 55.29 (SD \pm 64.30 ). After washing, the contamination level was reduced to an average of $3.09(\mathrm{SD} \pm 5.04) \mathrm{CFU}$ per $25 \mathrm{~cm}^{2}$ for prints in Group A. In Group B, the average CFU was reduced to 1.47 $(\mathrm{SD} \pm 4.77)$ per $25 \mathrm{~cm}^{2}$. As presented in Table 2, there was no significant difference in the average contamination degree between the two groups before washing ( $p>0.05)$, but there was a significant difference between the two groups after washing $(\mathrm{p}<0.05)$.

\section{Reduction in potentially pathogenic bacteria after washing}

As presented in Table 3, comparison of the average CFU per $25 \mathrm{~cm}^{2}$ in the groups before and after washing showed a reduction of general, potentially pathogenic bacteria of $96.25 \%$ for the 45 prints in Group A where the uniforms were washed without a disinfectant detergent. Moreover, an average reduction of $97.35 \%$ was found for the 45 prints in Group B where the uniforms were washed using a disinfectant detergent. The difference between the CFU reductions in the groups came close to significance $(\mathrm{p}=0.06)$.

\section{General prevalence}

As demonstrated in Table 4, Pseudomonas and E. coli were not found on any of the prints in the study. Out of 90 prints, we found $S$. aureus on $21.11 \%$ (CI 95\% \pm 7.80 ), B. cereus on $27.78 \%$ (CI 95\% \pm 9.80 ) and Enterococcus as well as Clostridium on $2.22 \%$ (CI $95 \% \pm 1.96)$.

\section{Prevalence before and after washing}

As presented in Table 5, the prevalence rates of the specific microorganisms were distributed differently after random selection. S. aureus was verified on $24.40 \%$ of the prints in Group A and on only $17.80 \%$ of the prints

Table 2 Degree of general potentially pathogenic contamination

\begin{tabular}{llll}
\hline CFU per 25 cm2 & Group A & Group B & p-value* \\
\hline$n$ & 45 & 45 & 0.06 \\
Mean & 82.49 & 55.29 & \\
Before washing & & & \\
SD & 65.21 & 64.30 & \\
Minimum & 0.00 & 0.00 & \\
Maximum & 200.00 & 200.00 & 0.02 \\
Cl 95\% & 19.59 & 19.32 & \\
After washing & & & \\
Mean & 3.09 & 1.47 & \\
SD & 5.04 & 4.77 & \\
Minimum & 0.00 & 0.00 & \\
Maximum & 29.00 & 1.43 & \\
Cl 95\% & 1.52 & &
\end{tabular}

*Student's t-test. 
Table 3 Reduction in general potentially pathogenic contamination after washing

\begin{tabular}{llllll}
\hline $\begin{array}{l}\text { CFU per } \\
\mathbf{2 5} \mathbf{~ c m}^{\mathbf{2}}\end{array}$ & $\mathbf{n}$ & $\begin{array}{l}\text { Before } \\
\text { washing }\end{array}$ & $\begin{array}{l}\text { After } \\
\text { washing }\end{array}$ & Reduction (\%) & p-value* \\
\hline Group A & 45 & 82.49 & 3.09 & $79.40(96.25)$ & 0.06 \\
Group B & 45 & 55.29 & 1.47 & $53.83(97.35)$ & \\
\hline
\end{tabular}

*Proportion tests.

in Group B $(\mathrm{p}>0.05)$. The prevalence of B. cereus was $15.60 \%$ in Group A and $40 \%$ in Group B. This difference between the two groups was significant $(\mathrm{p}<0.05)$. Enterococcus was verified on $4.40 \%$ of uniform prints in Group A and on 0\% of the prints in Group B Clostridium was not found on any of the prints in Group A, but the prevalence of this bacterium was $4.40 \%$ on the 45 uniform prints in Group B.

As shown in Table 6, only two prints were positive for $S$. aureus among the 45 prints in Group A after washing, which equals a prevalence of $4.4 \%$ (CI 95\% \pm 6.1 ) for uniforms washed without disinfecting detergent. In Group B, no prints tested positive to $S$. aureus. None of the 90 prints divided into Group A and Group B tested positive to Pseudomonas, E. coli, B. cereus, Enterococcus or Clostridium after washing (p was not calculated due to non-prevalence).

\section{Discussion}

Several healthcare related studies have found that healthcare-related uniforms were contaminated with various potentially pathogenic bacteria and thus associated with a risk of patient infection $[5,10,11]$. However, no Danish study has previously examined the bacterial contamination of prehospital uniforms which leaves us with no results for comparison. However, since our results indicate that an average print from a prehospital uniform has similar or fewer potentially pathogenic bacterial colonies than found on uniforms from in-hospital studies [12], we suggest that the contamination level of uniforms in prehospital settings is, to some extent, comparable to the contamination level of in- hospital uniforms.

Our findings of potentially pathogenic bacteria correspond to previous findings in hospital-based studies $[1,12]$. Our results indicate that S. aureus, Enterococcus and Clostridium are present on used prehospital uniforms with a presumed bacterial susceptibility comparable to

Table 4 Prevalence before washing all uniforms $(n=90)$

\begin{tabular}{lllll}
\hline & Number & $\%$ & SE & Cl 95\% \\
\hline S. aureus & 19 & 21.11 & 0.04 & \pm 7.80 \\
B. cereus & 25 & 27.78 & 0.05 & \pm 9.80 \\
Enterococcus & 2 & 2.22 & 0.01 & \pm 1.96 \\
Clostridium & 2 & 2.22 & 0.01 & \pm 1.96 \\
\hline
\end{tabular}

Table 5 Prevalence of specific bacteria before washing, grouped $(n=45)$

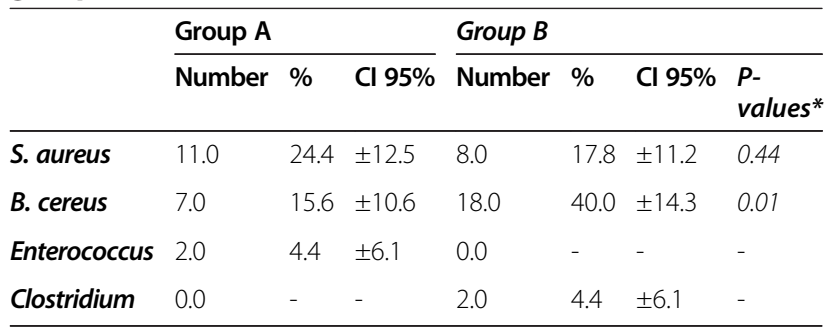

${ }^{*}$ Chi-squared tests.

that of patient isolates; however, we did not collect patients' samples and therefore cannot compare the results to those of patient isolates. Nevertheless, the present bacteria are already known to cause healthcare associated infections (HAIs).

The fact that we found a lower prevalence of Enterococcus than in a previous study focusing on nurse uniforms [4] is probably explained by the fact that nurses' tasks are not identical to those performed by staff in the prehospital setting. For example, prehospital staff may perform much fewer tasks related to personal care, e.g. hygiene following a restroom visit, etc., which are known to be associated with infection. Although we did not find E. coli or Pseudomonas, we cannot dismiss that prehospital uniforms could be contaminated with either of these microorganisms. Nevertheless, we assume that the contamination level and hence the risk of infecting patients is, indeed, limited.

Washing and tumble-drying is known to aid bacterial reduction, especially as prehospital uniforms dictate a maximum washing temperature of $60 \mathrm{C}$. However, our results show that washing and tumble-drying in domestic machines for 1 hour, regardless of the choice of washing powder, did not eliminate all microorganisms. The washing machine, the tumble-dryer and the environment could all have caused this contamination, and it would have been beneficial if we had collected environmental samples which would have provides us with knowledge of a possible correlation between contamination of uniforms and contamination of machines and/or the environment. In addition, we could also have tested the temperature in the tumble dryer and the washing machine.

Unfortunately, our methodology has a number of limitations that must be addressed. Firstly, the results of the washing process are compromised given that the two

Table 6 Prevalence of specific bacteria after washing, grouped $(n=45)$

\begin{tabular}{|c|c|c|c|c|c|c|}
\hline & \multicolumn{3}{|l|}{ Group A } & \multicolumn{3}{|l|}{ Group B } \\
\hline & Number & $\%$ & $\mathrm{Cl} 95 \%$ & Number & $\%$ & $\mathrm{Cl} 95 \%$ \\
\hline S. aureus & 2.0 & 4.4 & \pm 6.1 & 0.0 & - & - \\
\hline
\end{tabular}


groups compared did not have the same degree of contamination at baseline. However, we were unable to ensure this as this was an in situ study. Secondly, due to practicalities such as timeframe and financial limitations, we did not perform a sample size calculation, which could have ensured a sample size sufficiently large to obtain a significant difference. Therefore, we acknowledge that this study should be considered a pilot study, and that a larger future study requires further consideration.

There is also a risk of bias associated with the position of the prints before and after washing. Although we tried to ensure that prints were taken from the same location before and after washing, there is a margin of error associated with the outcome as we cannot guarantee that the prints were placed in the same location before and after washing. Another important issue that must be emphasized is the variation in our results caused by the use of domestic washing machines; however, we did intend to illustrate the result during the practical laundering and handling process in the prehospital setting. We used three uniform sampling locations which have previously been shown to be the most contaminated locations for assessing the mean degree of contamination of the uniforms, and we assessed the mean degree of contamination. The sampling technique itself was well known by the environmental laboratory performing the analysis, and its feasibility for that purpose is well-established in current literature. It was therefore decided not to further test its reproducibility.

As mentioned, the number of international studies on the effect of different washing methods is very limited $[7,10]$, and no previous studies in the Danish ambulance services can substantiate our findings on bacterial contamination or on the quality of the used washing methods. At the same time, evidence of the risk associated with the transmission of microorganisms between staff and patients is very limited. Furthermore, no Danish correlation studies between bacterial CFU and incidence of infection in the prehospital setting have been performed. Nor have any studies sought to correlate different methods of washing with the incidence of infections. In Denmark, no comprehensive registration of HAIs is presently in place and therefore such analysis is not possible. We recommend that such registration be introduced to enable studies on the association between contamination degree and risk of infection.

Overall, further studies are needed on prehospital bacterial contamination and hygiene interventions. Increased evidence of contamination degrees, their underlying causes and the effect of hygiene interventions and procedures will, all things being equal, strengthen infection control in the prehospital setting.

\section{Conclusion}

The results of this study indicate the presence of a contamination level which - in several respects - underpins the need to comply with existing hygiene instructions in the prehospital setting. Daily change of uniform, washing at a minimum of 60 degrees Celsius and use of a disinfectant detergent (acetic peroxide) reduce the degree of bacterial contamination and thus reduce the risk that patients become infected. The results concerning a potentially pathogenic contamination level and the prevalence of specific microorganisms, including B. cereus, Clostridium, $S$. aureus and Enterococcus, stress the need for daily change of uniform and a high hygiene standard in daily prehospital work with patients to avoid increasing the risk that microorganisms are transmitted from ambulance uniforms to patients.

\section{Competing interests}

Heidi Storm Vikke conducted this study during an internship forming part of her Master of Health Science at the University of Southern Denmark, SDU.

The ambulance staff employed by Falck Denmark A/S, the organization in which the author was an intern, wore the uniforms collected and examined in the study. Furthermore, at the time of publication of this article, the author Heidi Storm Vikke is a fulltime scientific research consultant at Falck Denmark A/S; and the co-author, Matthias Giebner, is a medical director in Falck Denmark A/S.

\section{Authors' contributions}

HSV and MG both participated in the design of the study. HSV and MG conceived of the study and both participated in its design and coordination and co-operated to draft the manuscript. HSV solely performed all statistical analyses. Both authors have read and approved the final manuscript.

\section{Acknowledgements}

The authors would like to thank the staff of Falck Denmark A/S for their dedication and participation in the provision of the uniforms included in the study.

Also, we would like to express our gratitude to Professor Jørgen T. Lauridsen, University of Southern Denmark (SDU), for providing a number of practical considerations that helped shape this article.

\section{Funding}

Falck Denmark A/S has defrayed all study costs.

Received: 3 November 2014 Accepted: 13 March 2015

Published online: 25 March 2015

\section{References}

1. Treakle AM, Thom KA, Furuno JP, Strauss SM, Harris AD, Perencevich EN Bacterial contamination of health care workers' white coats. Am J Infect Control. 2009:37(2):101-5.

2. Burden M, Keniston A, Frank MG, Brown CA, Zoucha J, Cervantes $L$, et al. Bacterial contamination of healthcare workers' uniforms: a randomized controlled trial of antimicrobial scrubs. J Hosp Med. 2013;8(7):380-5.

3. Munoz-Price LS, Arheart KL, Lubarsky DA, Birnbach DJ. Differential laundering practices of white coats and scrubs among health care professionals. Am J Infect Control. 2013;41(6):565-7.

4. Wiener-Well Y, Galuty M, Rudensky B, Schlesinger Y, Attias D, Yinnon AM. Nursing and physician attire as possible source of nosocomial infections. Am J Infect Control. 2011:39(7):555-9.

5. Hambraeus A. Transfer of Staphylococcus aureus via nurses' uniforms. J Hyg. 1973:71(4):799-814

6. Lankford MG, Collins S, Youngberg L, Rooney DM, Warren JR, Noskin GA. Assessment of materials commonly utilized in health care: implications for bacterial survival and transmission. Am J Infect Control. 2006;34(5):258-63. 
7. Wilson JA, Loveday HP, Hoffman PN, Pratt RJ. Uniform: an evidence review of the microbiological significance of uniforms and uniform policy in the prevention and control of healthcare-associated infections. Report to the Department of Health (England). J Hosp Infect. 2007;66(4):301-7.

8. Furuno JP, Perencevich EN, Johnson JA, Wright MO, McGregor JC, Morris JG, et al. Methicillin-resistant Staphylococcus aureus and vancomycin-resistant Enterococci co-colonization. Emerg Infect Dis. 2005;1 1(10):1539-44.

9. Perry C, Marshall R, Jones E. Bacterial contamination of uniforms. J Hosp Infect. 2001;48(3):238-41.

10. Hambraeus A, Ransjo U. Attempts to control clothes-borne infection in a burn unit I. Experimental investigations of some clothes for barrier nursing. J Hyg. 1977;79(2):193-202.

11. Hedin G. Staphylococcus epidermidis-hospital epidemiology and the detection of methicillin resistance. Scand J Infect Dis Suppl. 1993;90:1-59.

12. Nordstrom JM, Reynolds KA, Gerba CP. Comparison of bacteria on new, disposable, laundered, and unlaundered hospital scrubs. Am J Infect Control. 2012:40(6):539-43.

\section{Submit your next manuscript to BioMed Central and take full advantage of:}

- Convenient online submission

- Thorough peer review

- No space constraints or color figure charges

- Immediate publication on acceptance

- Inclusion in PubMed, CAS, Scopus and Google Scholar

- Research which is freely available for redistribution 